\title{
On The Fractional Derivative Formulae Involving the Product of A General Class Of Polynomials And The Multivariable $A$ - Function
}

\author{
Yashwant Singh $^{1}$, Harmendra Kumar Mandia ${ }^{2}$ \\ ${ }^{I}$ Department of Mathematics, Government College, Kaladera, Jaipur (Rajasthan), India \\ 2. Department of Mathematics, Seth Motilal (P.G.) College, Jhunjhunu, Rajasthan, India
}

\begin{abstract}
In the present paper, we obtain three fractional derivative formulae (FDF). The first involves the product of a general class of polynomials and the multivariable A-function. The second of a general class of polynomials and two multivariable $A$-functions and has been obtained with the help of the generalized Leibnitz rule for fractional derivatives. The last FDF also involves the product of a general class of polynomials and the multivariable A-function but it is obtained by the application of the first FDF twice and it involves two independent variables instead of one. Two polynomials and the functions involved in all our fractional
\end{abstract} derivative formulae as well as their arguments which are of the type $x^{\rho} \prod_{t=1}^{s}\left(x^{t_{i}}+\alpha_{i}\right)^{\sigma_{i}}$ are quite general in nature. These formulae, besides being of very general character have been put in a compact form avoiding the occurrence of infinite series and thus making them useful in applications. Our findings provide interesting unifications and extensions of a number of (new and known) results. For the sake of illustration, we give here exact references to the results to the results (in essence) of six research papers [3,4,12,13,14,15] that follow as particular cases of our findings. In the end, we record a new fractional derivative formula involving the product of the Hermite polynomials and the product of $r$ different Whittakar functions as a simple special case of our first formula.

Key words: Reimann-Liouville and Erdelyi-Kober fractional operatore, Fractional derivative formulae, General class of polynomials, Multivariable A-function, Generalized Leibnitz rule.

\section{Introduction}

We shall define the fractional integrals and derivatives of a function $f(x)$ ([12], pp.528-529) (see also $[7,8,10])$ as follows:

Let $\alpha, \beta$ and $\gamma$ be complex numbers. The fractional integral $(\operatorname{Re}(\alpha)>0)$ and derivative $(\operatorname{Re}(\alpha)<0$ of a function $f(x)$ defined on $(0, \infty)$ is given by

$I_{o, x}^{\alpha, \beta, \gamma} f(x)=\left\{\begin{array}{l}\frac{x^{-\alpha-\beta}}{\Gamma(\alpha)} \int_{0}^{x}(x-t)^{\alpha-1} F\left(\alpha+\beta-\gamma ; \alpha ; 1-\frac{t}{x}\right) f(t) d t,(\operatorname{Re}(\alpha)>0, \\ \frac{d^{q}}{d x^{q}} I_{o, x}^{\alpha+q, \beta-q, \gamma-q} f(x),(\operatorname{Re}(\alpha) \leq 0,0<\operatorname{Re}(\alpha)+q \leq 1 ; q=1,2,3, \ldots),\end{array}\right.$

Where $F$ is the gauss hypergeometric function.

The operator $A$ includes both the Riemann-Liouville and Erdelyi-Kober fractional operators as follows:

The Riemann-Liouville operator

$R_{o, x}^{\alpha} f(x)=\left\{\begin{array}{l}R_{o, x}^{\alpha,-\alpha, \gamma} f(x)=\frac{1}{\Gamma(\alpha)} \int_{0}^{x}(x-t)^{\alpha-1} f(t) d t,(\operatorname{Re}(\alpha)>0, \\ \frac{d^{q}}{d x^{q}} R_{o, x}^{\alpha+q} f(x),(\operatorname{Re}(\alpha) \leq 0,0<\operatorname{Re}(\alpha)+q \leq 1 ; q=1,2,3, \ldots), \quad \text { The Erdelyi-Kober operator }\end{array}\right.$

$E_{0, x}^{\alpha, \gamma} f(x)=I_{0, x}^{\alpha, \alpha, \gamma} f(x)=\frac{x^{-\alpha-\gamma}}{\Gamma(\alpha)} \int_{0}^{x}(x-t)^{\alpha-1} t^{\gamma} f(t) d t, \operatorname{Re}(\alpha)>0$,

Also, $S_{n}^{m}[x]$ occurring in the sequel denotes the general class of polynomials introduced by Srivastava ([13],p.1, eq. (1)) 
$S_{n}^{m}[x]=\sum_{k=0}^{[n / m]} \frac{(-n)_{m k}}{k !} A_{n, k} x^{k}, n=0,1,2, \ldots$

Where $m$ is an arbitrary positive integer and the coefficients $A_{n, k}(n, k \geq 0)$ are arbitrary constants, real or complex. On suitably specializing the coefficients $A_{n, k}, S_{n}^{m}[x]$ yields a number of known polynomials as its special cases. These include, among others, the Hermite polynomials, the Jacobi polynomials, the Leguerre polynomials, the Bessel polynomials, the gould-Hopper polynomials, the Brafman polynomials and several others ([18],pp.158-161).

The multivariable $A$-function introduced by Gautam et.al. [2] will be define and represent it in the following manner :

$$
\begin{aligned}
& A\left[z_{1}, \ldots, z_{r}\right]=A_{p, q:\left(p_{1}, q_{1}\right) \ldots:\left(p_{r}, q_{r}\right)}^{m, n:\left(m_{1}, n_{r}\right):\left(m_{r}, n\right)} \\
& {\left[\begin{array}{l|l}
z_{1}, \ldots, z_{r} & \begin{array}{l}
\left(a_{j}, A_{j}^{\prime}, \ldots, A_{j}^{(r)}\right)_{1, p}:\left(a_{j}^{\prime}, \alpha_{j}^{\prime}\right)_{1, p^{\prime}} \ldots .\left(a_{j}^{(r)}, \alpha_{j}^{(r)}\right)_{1, p}^{(r)} \\
\left(b_{j}, B_{j}^{\prime}, \ldots, B_{j}^{(r)}\right)_{1, q}:\left(b_{j}^{\prime}, \beta_{j}^{\prime}\right)_{1, q} \div \ldots\left(b_{j}^{(r)}, \beta_{j}^{(r)}\right)_{1, q}^{(r)}
\end{array}
\end{array}\right]} \\
& =\frac{1}{(2 \pi w)^{r}} \int_{L_{1}} \ldots \int_{L_{r}} \phi_{1}\left(s_{1}\right) \ldots \phi_{r}\left(s_{r}\right) \psi\left(s_{1}, \ldots, s_{r}\right) z_{1}^{s_{1}}, \ldots, z_{r}^{s_{r}} d s_{1} \ldots d s_{r}
\end{aligned}
$$

Where

$$
\begin{gathered}
w=\sqrt{(-1)} \\
\phi_{i}\left(s_{i}\right)=\frac{\prod_{j=1}^{m_{i}} \Gamma\left(b_{j}^{(i)}-\beta_{j}^{(i)} s_{i}\right) \prod_{j=1}^{n_{i}} \Gamma\left(1-a_{j}^{(i)}+\alpha_{j}^{(i)} s_{i}\right)}{\prod_{j=m_{i}+1}^{q_{i}} \Gamma\left(1-b_{j}^{(i)}+\beta_{j}^{(i)} s_{i}\right) \prod_{j=n_{i}+1}^{p_{i}} \Gamma\left(a_{j}^{(i)}-\alpha_{j}^{(i)} s_{i}\right)} \forall i \in(1,2, \ldots, r) \\
\psi\left(s_{1}, \ldots, s_{r}\right)=\frac{\prod_{j=1}^{n} \Gamma\left(1-a_{j}+\sum_{i=1}^{r} A_{j}^{(i)} s_{i}\right) \prod_{j=1}^{m} \Gamma\left(b_{j}-\sum_{i=1}^{r} B_{j}^{(i)} s_{i}\right)}{\prod_{j=n+1}^{p} \Gamma\left(a_{j}-\sum_{i=1}^{r} A_{j}^{(i)} s_{i}\right) \prod_{j=m+1}^{q} \Gamma\left(1-b_{3 j}+\sum_{i=1}^{r} B_{j}^{(i)} s_{i}\right)}
\end{gathered}
$$

$\alpha_{j}^{(i)}, \beta_{j}^{(i)}, \alpha_{j}^{(i)}, \beta_{j}^{(i)}(i=1, \ldots, r)$ are positive numbers, $a_{j}^{(i)}, b_{j}^{(i)}, a_{j}, b_{j}(i=1, \ldots, r)$ are complex numbers and here $m_{i}, n_{i}, p_{i}, q_{i}(i=1, \ldots, r)$ are non-negative integers where $0 \leq m_{i} \leq q_{i}, 0 \leq n_{i} \leq p_{i}$. Here $(i)$ denotes the numbers of dashes. The contours $L_{i}$ in the complex $s_{i}$-plane is of the Mellin-Barnes type which runs from $-w \infty 0$ to $+w \infty$ with indentations, if necessary, to ensure that all the poles of $\Gamma\left(b_{j}^{(i)}-\beta_{j}^{(i)} s_{i}\right)\left(j=1, \ldots, m_{i}\right)$ are separated from those of $\Gamma\left(1-a_{j}+\sum_{i=1}^{r} \alpha_{j}^{(i)} s_{i}\right)\left(j=1, \ldots, n_{r}\right)$

For further details and asymptotic expansion of the $A$-function one can refer by Gautam et.al. [2]. In what follows, the multivariable $A$-function defined by [2] will be represented in the contracted notation:

$$
A_{p, q:\left(p_{1}, q_{1}\right) \cdots\left(p_{r}, q_{r}\right)}^{m, n:\left(m_{1}, n_{1}\right) \cdots\left(m_{r}, n_{r}\right)}\left[z_{1}, \ldots, z_{r}\right]
$$

Or simply by $A\left[z_{1}, \ldots z_{r}\right]$.

\subsection{Fractional Derivative Formula 1:}

\section{Main Results}

$$
\begin{aligned}
& I_{0, x}^{\alpha, \beta, \gamma}\left\{x^{\rho} \prod_{i=1}^{s}\left(x^{t_{i}}+\alpha_{i}\right)^{\sigma_{i}} \prod_{j=1}^{t} S_{n_{j}}^{m_{j}}\left[e_{j} x^{\lambda_{j}} \prod_{i=1}^{s}\left(x^{t_{i}}+\alpha_{i}\right)^{\eta_{j}^{(i)}}\right]\right. \\
& \left.A\left[z_{1} x^{u_{1}} \prod_{j=1}^{s}\left(x^{t_{i}}+\alpha_{i}\right)^{-v_{i}^{\prime}}, \ldots, z_{r} x^{u_{1}} \prod_{j=1}^{s}\left(x^{t_{i}}+\alpha_{i}\right)^{-v_{i}^{(r)}}\right]\right\}
\end{aligned}
$$




$$
\begin{aligned}
& =\alpha_{1}^{\sigma_{1}} \ldots \alpha_{s}^{\sigma_{s}} x^{\rho-\beta} \sum_{k_{1}=0}^{\left[n_{1} / m_{1}\right]} \ldots \sum_{k_{1}=0}^{\left[n_{1} / m_{1}\right]} \frac{\left(-n_{1}\right)_{m_{1} k_{1}} \ldots\left(-n_{t}\right)_{m_{t} k_{t}}}{k_{1} ! \ldots k_{t} !} A_{n_{1}, k_{1}}^{\prime} \ldots A_{n_{t}, k_{t}}^{(t)} \\
& e_{1}^{k_{1}} \ldots e_{t}^{k_{t}} \alpha_{1}^{\eta_{1} k_{1}+\ldots+\eta_{1}^{(t)} k_{t}} \ldots \alpha_{s}^{\eta_{s} k_{1}+\ldots+\eta_{s}^{(t)} k_{t}} x^{\lambda_{1} k_{1}+\ldots+\lambda_{t} k_{t}}
\end{aligned}
$$

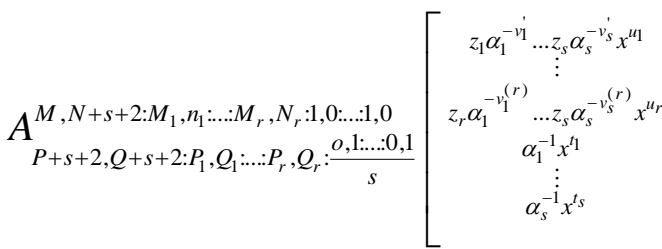

$$
\begin{aligned}
& \left(a_{j} ; A_{t_{j}}^{\prime}, \ldots, A_{j}^{(r)}, \frac{0, \ldots, 0}{s}\right)_{1, P} ;\left(-\rho-\lambda_{1} k_{1}-\ldots-\lambda_{t} k_{t} ; u_{1}, \ldots, u_{r}, t_{1}, \ldots, t_{s}\right), \\
& \left(b_{j} ; B_{j}^{\prime}, \ldots, B_{j}^{(r)}, \frac{0, \ldots, 0}{s}\right)_{1, Q} ;\left(\beta-\rho-\lambda_{1} k_{1}-\ldots-\lambda_{t} k_{t} ; u_{1}, \ldots, u_{r}, t_{1}, \ldots, t_{s}\right), \\
& \left(-\beta-\gamma-\rho-\lambda_{1} k_{1}-\ldots-\lambda_{t} k_{t} ; u_{1}, \ldots, u_{r}, t_{1}, \ldots, t_{s}\right),\left(1+\sigma_{1}+\eta_{1}^{\prime} k_{1}+\ldots+\eta_{1}^{(t)} k_{t} ; v_{1}^{\prime}, \ldots, v_{1}^{(r)}, 1, \frac{0, \ldots, 0}{s-1}\right), \ldots, \\
& \left(-\alpha-\gamma-\rho-\lambda_{1} k_{1}-\ldots-\lambda_{t} k_{t} ; u_{1}, \ldots, u_{r}, t_{1}, \ldots, t_{s}\right),\left(1+\sigma_{1}-\eta_{1}^{\prime} k_{1}+\ldots+\eta_{1}^{(t)} k_{t} ; v_{1}^{\prime}, \ldots, v_{1}^{(r)}, \frac{0, \ldots, 0}{s}\right), \ldots, \\
& \left(1+\sigma_{s}+\eta_{s}^{\prime} k_{1}+\ldots+\eta_{s}^{(t)} k_{t} ; v_{s}^{\prime}, \ldots, v_{s}^{(r)}, 1, \frac{0, \ldots, 0}{s-1}\right):\left(c_{j}^{\prime}, \gamma_{j}^{\prime}\right)_{1, P} ; \ldots ;\left(c_{j}^{(r)}, \gamma_{j}^{(r)}\right)_{1, P} \\
& \left(1+\sigma_{s}-\eta_{s}^{\prime} k_{1}+\ldots+\eta_{s}^{(t)} k_{t} ; v_{s}^{\prime}, \ldots, v_{s}^{(r)}, \frac{0, \ldots, 0}{s}\right) \text { : } \\
& \begin{array}{l}
: \ldots: \\
\left.\left(d_{j}^{\prime}, \delta_{j}^{\prime}\right)_{1, Q_{1}} ; \ldots ;\left(d_{j}^{(r)}, \delta_{j}^{(r)}\right)_{1, Q_{r}} ; \frac{(0,1), \ldots,(0,1)}{s}\right]
\end{array}
\end{aligned}
$$

Provided that

(i) $\operatorname{Re}(\alpha)>0$, the quantities $t_{1}, \ldots, t_{s}, \lambda_{1} \eta_{1}^{\prime}, \ldots, \lambda_{t} \eta_{1}^{(t)}, \ldots, \lambda_{1} \eta_{s}^{\prime}, \ldots, \lambda_{t} \eta_{s}^{(t)}, u_{1}, v_{1}^{\prime}, \ldots, v_{s}^{\prime}, u_{r}, v_{1}^{(r)}, \ldots, v_{s}^{(r)}$ are all positive (some of them may however decrease to zero provided that the resulting integral has a meaning),

(ii) $\operatorname{Re}(\rho)+\sum_{i=1}^{r} u_{i} \min _{1 \leq j \leq M_{i}}\left[\operatorname{Re}\left(\frac{d_{j}^{(i)}}{\delta_{j}^{(i)}}\right)\right]+1>0$

Also the number occurring below the line at any place on the right-hand side of (8) and throughout the paper indicates the total number of zeros/ones/pairs covered by it. Thus $\frac{0, \ldots, 0}{r}, \frac{1, \ldots, 1}{r}, \frac{0,1 ; \ldots ; 0,1}{r}$ would mean $\mathrm{r}$ zeros/r ones/r pairs, and so on.

\subsection{Fractional Derivative Formula 2:}

$$
\begin{aligned}
& I_{0, x}^{\alpha, \beta, \gamma}\left\{x^{\rho} \prod_{i=1}^{s}\left(x^{t_{i}}+\alpha_{i}\right)^{\sigma_{i}} \prod_{j=1}^{t} S_{n_{j}}^{m_{j}}\left[e_{j} x^{\lambda_{j}} \prod_{i=1}^{s}\left(x^{t_{i}}+\alpha_{i}\right)^{\eta_{j}^{(i)}}\right]\right. \\
& A\left[z_{1} x^{u_{1}} \prod_{j=1}^{s}\left(x^{t_{i}}+\alpha_{i}\right)^{-v_{i}^{\prime}}, \ldots, z_{r} x^{u_{1}} \prod_{j=1}^{s}\left(x^{t_{i}}+\alpha_{i}\right)^{-v_{i}^{(r)}}\right] \\
& \left.A^{*}\left[z_{r+1} x^{u_{r}+1} \prod_{j=1}^{s-1}\left(x^{t_{i}}+\alpha_{i}\right)^{-v_{i}^{(r+1)}}, \ldots, z_{r+r} x^{u_{r+r}} \prod_{j=1}^{s-1}\left(x^{t_{i}}+\alpha_{i}\right)^{-v_{i}^{(r+r)}}\right]\right\} \\
& =\alpha_{1}^{\sigma_{1}} \ldots \alpha_{s}^{\sigma_{s}} x^{\rho-\beta} \sum_{t=0}^{\infty} \sum_{k_{1}=0}^{\left[n_{1} / m_{1}\right]} \ldots \sum_{k_{1}=0}^{\left[n_{1} / m_{1}\right]}\left(\begin{array}{l}
-\beta \\
t
\end{array}\right) \frac{\left(-n_{1}\right)_{m_{1} k_{1}} \ldots\left(-n_{t}\right)_{m_{t} k_{t}}}{k_{1} ! \ldots k_{t} !} A_{n_{1}, k_{1}}^{\prime} \ldots A_{n_{t}, k_{t}}^{(t)} \\
& e_{1}^{k_{1}} \ldots e_{t}^{k_{t}} \alpha_{1}^{\eta_{1} k_{1}+\ldots+\eta_{1}^{(t)} k_{t}} \ldots \alpha_{s}^{\eta_{s}^{k_{1}+\ldots+}+\eta_{s}^{(t)} k_{t}} x^{\lambda_{1} k_{1}+\ldots+\lambda_{t} k_{t}} \\
& \begin{array}{l}
A^{M, N+N+2 s+3: M_{1}, N_{1}: \ldots: M_{r}, N_{r}: 1,0: \ldots .1,0: M_{r+1}, N_{r+1} ; \ldots, M_{r+\tau}, N_{r+\tau} ; 1,0 ; \ldots, 1,0} \\
\quad P+P+2 s+3, Q+Q+2 s+3: P_{1}, Q_{1}: \ldots .: P_{r}, Q_{r}: \frac{o, 1: \ldots: 0,1}{s} ; P_{r+1}, Q_{r+1}, \ldots, P_{r+\tau}, Q_{r+\tau} ; \frac{o, 1 \ldots .: 0,1}{s}
\end{array}
\end{aligned}
$$




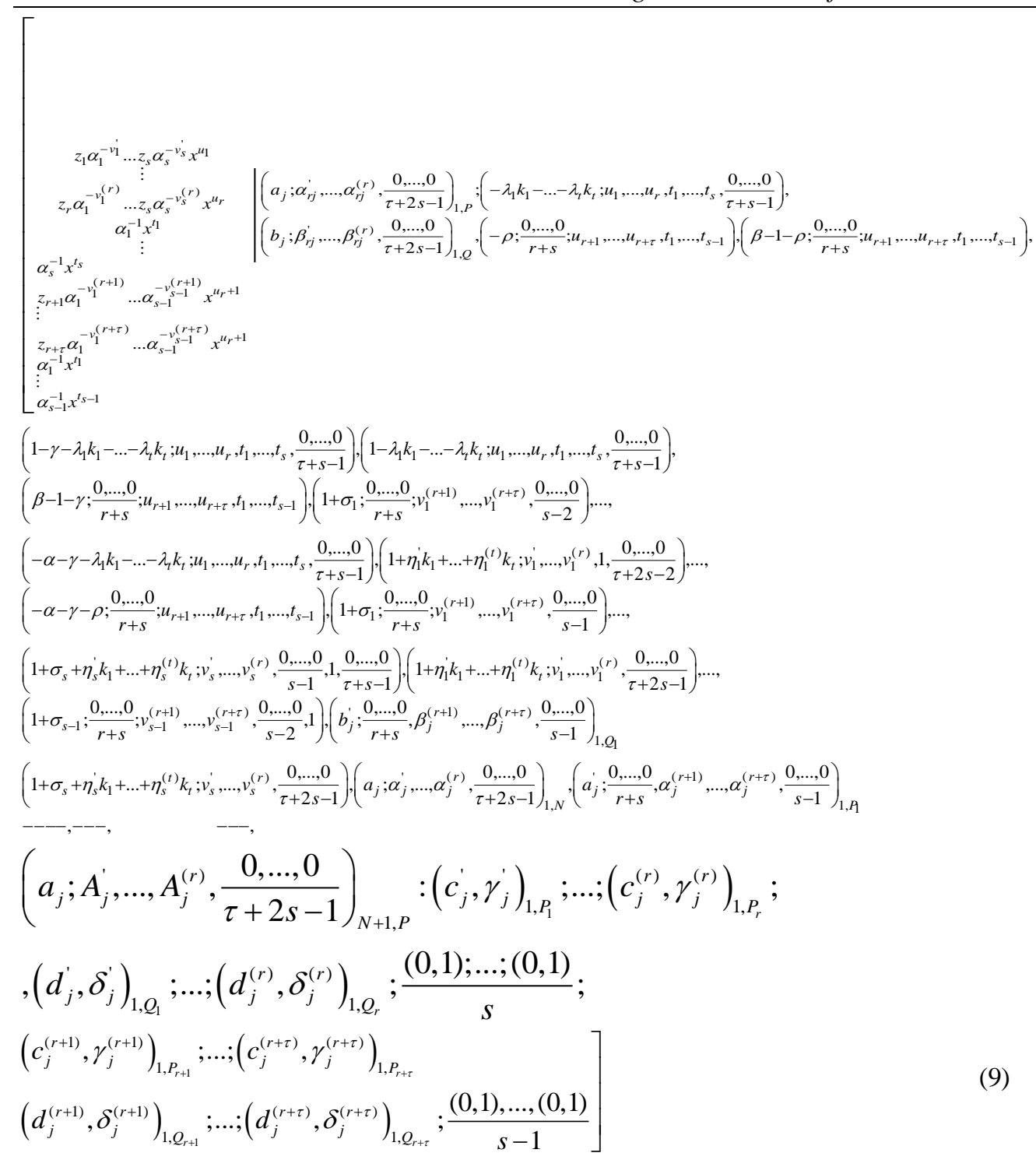

Here $A *\left[z_{r+1}, \ldots, z_{r+\tau}\right]$ stands for the following multivariable $A$-function of $\tau$ complex variables $z_{r+1}, \ldots, z_{r+\tau}$.

$A\left[z_{1}, \ldots, z_{r}\right]=I_{p^{\prime}, q^{\prime}:\left(p_{r+1}, q_{r+1}\right) \cdots:\left(p_{r+\tau}, q_{r+\tau}\right)}^{\left.m_{r+\tau}\right)}$

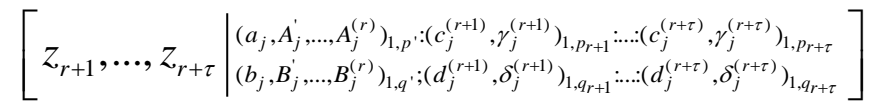

The function occurring on the right hand side of (9) is the $A$-function of $r+2 s+\tau-1$ variables provided that (i) $\operatorname{Re}(\alpha)>0$, the quantities $t_{1}, \ldots, t_{s}, \lambda_{1} \eta_{1}^{\prime}, \ldots, \lambda_{t} \eta_{1}^{(t)}, \ldots, \lambda_{1} \eta_{s}^{\prime}, \ldots, \lambda_{t} \eta_{s}^{(t)}, u_{1}, v_{1}^{\prime}, \ldots, v_{s}^{\prime}, u_{r}, v_{1}^{(r)}, \ldots, v_{s}^{(r)}, u_{r+1}, v_{1}^{(r+1)}, \ldots, v_{s-1}^{(r+1)}, u_{r+r}, v_{1}^{(r+\tau)}, \ldots, v_{s-1}^{(r+\tau)}$ are all positive (some of them may however decrease to zero provided that the resulting integral has a meaning),

(ii) $\operatorname{Re}(\rho)+\sum_{i=1}^{r+\tau} u_{i} \min _{1 \leq j \leq M_{i}}\left[\operatorname{Re}\left(\frac{d_{j}^{(i)}}{\delta_{j}^{(i)}}\right)\right]+1>0$

\subsection{Fractional Derivative Formula 3:}

$$
\begin{aligned}
& I_{0, x}^{\alpha, \beta, \gamma} I_{0, y}^{\alpha^{\prime}, \beta^{\prime}, \gamma^{\prime}}\left\{x^{\rho} y^{\rho^{\prime}} \prod_{i=1}^{s}\left(x^{t_{i}}+\alpha_{i}\right)^{\sigma_{i}}\left(y^{t_{i}^{\prime}}+\beta_{i}\right)^{\sigma_{i}^{\prime}} \prod_{j=1}^{t} S_{n_{j}}^{m_{j}}\left[e_{j} x^{\lambda_{j}} y^{\varsigma_{j}} \prod_{i=1}^{s}\left(x^{t_{i}}+\alpha_{i}\right)^{\eta_{j}^{(i)}}\left(y^{t_{i}}+\beta_{i}\right)^{\tau_{j}^{(i)}}\right]\right. \\
& \left.A\left[z_{1} x^{u_{1}} y^{u_{i}} \prod_{j=1}^{s}\left(x^{t_{i}}+\alpha_{i}\right)^{-v_{i}}\left(y^{t_{i}^{\prime}}+\beta_{i}\right)^{-w_{i}}, \ldots, z_{r} x^{u_{1}} y^{u_{i}} \prod_{j=1}^{s}\left(x^{t_{i}}+\alpha_{i}\right)^{-v_{i}^{(r)}}\left(y^{t_{i}}+\beta_{i}\right)^{-w_{i}^{(r)}}\right]\right\}
\end{aligned}
$$




$$
\begin{aligned}
& =\alpha_{1}^{\sigma_{1}} \ldots \alpha_{s}^{\sigma_{s}} \beta_{1}^{\sigma_{1}^{\prime}} \ldots \beta_{s}^{\sigma_{s}^{\prime}} x^{\rho-\beta} y^{\rho^{\prime}-\beta^{\prime}} \sum_{k_{1}=0}^{\left[n_{1} / m_{1}\right]} \ldots \sum_{k_{t}=0}^{\left[n_{t} / m_{t}\right]} \frac{\left(-n_{1}\right)_{m_{1} k_{1}} \ldots\left(-n_{t}\right)_{m_{t} k_{t}}}{k_{1} ! \ldots k_{t} !} A_{n_{1}, k_{1}}^{\prime} \ldots A_{n_{t}, k_{t}}^{(t)} \\
& e_{1}^{k_{1}} \ldots e_{t}^{k_{t}} \alpha_{1}^{\eta_{1}^{\prime} k_{1}+\ldots+\eta_{1}^{(t)} k_{t}} \ldots \alpha_{s}^{\eta_{s}^{\prime} k_{1}+\ldots+\eta_{s}^{(t)} k_{t}} \beta_{1}^{\tau_{1}^{\prime} k_{1}+\ldots+\tau_{1}^{(t)} k_{t}} \ldots \beta_{s}^{\tau_{s}^{\prime} k_{1}+\ldots+\tau_{s}^{(t)} k_{t}} x^{\lambda_{1} k_{1}+\ldots+\lambda_{t} k_{t}} y^{\varsigma_{1} k_{1}+\ldots+\varsigma_{t} k_{t}}
\end{aligned}
$$

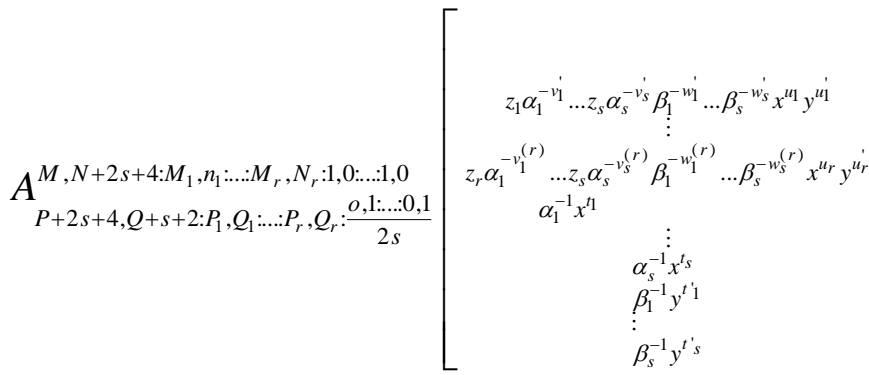

$$
\begin{aligned}
& \mid\left(a_{j} ; A_{j}^{\prime}, \ldots, A_{j}^{(r)}, \frac{0, \ldots, 0}{2 s}\right)_{1, P} ;\left(-\rho-\lambda_{1} k_{1}-\ldots-\lambda_{t} k_{t} ; u_{1}, \ldots, u_{r}, \frac{0, \ldots, 0}{s}, t_{1}, \ldots, t_{s}\right), \\
& \left(\left(b_{j} ; B_{j}^{\prime}, \ldots, B_{j}^{(r)}, \frac{0, \ldots, 0}{2 s}\right)_{1, Q} ;\left(\beta-\rho-\lambda_{1} k_{1}-\ldots-\lambda_{t} k_{t} ; u_{1}, \ldots, u_{r}, \frac{0, \ldots, 0}{s}, t_{1}, \ldots, t_{s}\right),\right. \\
& \left(1+\sigma_{s}+\eta_{s}^{\prime} k_{1}+\ldots+\eta_{s}^{(t)} k_{t} ; v_{s}^{\prime}, \ldots, v_{s}^{(r)}, 1, \frac{0, \ldots, 0}{2 s-1}\right):\left(c_{j}^{\prime}, \gamma_{j}^{\prime}\right)_{1, P_{1}} ; \ldots ;\left(c_{j}^{(r)}, \gamma_{j}^{(r)}\right)_{1, P_{r}} \\
& \left(1+\sigma_{s}-\eta_{s}^{\prime} k_{1}+\ldots+\eta_{s}^{(t)} k_{t} ; v_{s}^{\prime}, \ldots, v_{s}^{(r)}, \frac{0, \ldots, 0}{2 s}\right),\left(b_{j} ; \beta_{j}^{\prime}, \ldots, \beta_{j}^{(r)}, \frac{0, \ldots, 0}{s}\right)_{1, Q_{r}}: \\
& \left(-\beta-\gamma-\rho-\lambda_{1} k_{1}-\ldots-\lambda_{t} k_{t} ; u_{1}, \ldots, u_{r}, \frac{0, \ldots, 0}{s}, t_{1}, \ldots, t_{s}\right),\left(1+\sigma_{1}+\eta_{1}^{\prime} k_{1}+\ldots+\eta_{1}^{(t)} k_{t} ; v_{1}^{\prime}, \ldots, v_{1}^{(r)}, \frac{0, \ldots, 0}{s}, 1, \frac{0, \ldots, 0}{s-1}\right), \ldots \\
& \left(-\alpha-\gamma-\rho-\lambda_{1} k_{1}-\ldots-\lambda_{t} k_{t} ; u_{1}, \ldots, u_{r}, \frac{0, \ldots, 0}{s}, t_{1}, \ldots, t_{s}\right),\left(1+\sigma_{1}-\eta_{1}^{\prime} k_{1}+\ldots+\eta_{1}^{(t)} k_{t} ; v_{1}^{\prime}, \ldots, v_{1}^{(r)}, \frac{0, \ldots, 0}{2 s}\right), \ldots, \\
& \left(-\rho^{\prime}-\varsigma_{1} k_{1}-\ldots-\varsigma_{t} k_{t} ; u_{1}^{\prime}, \ldots, u_{r}^{\prime}, \frac{0, \ldots, 0}{s}, t_{1}^{\prime}, \ldots, t_{s}^{\prime}\right) \\
& \left(\beta^{\prime}-\rho^{\prime}-\varsigma_{1} k_{1}-\ldots-\varsigma_{t} k_{t} ; u_{1}^{\prime}, \ldots, u_{r}^{\prime}, \frac{0, \ldots, 0}{s}, t_{1}^{\prime}, \ldots, t_{s}^{\prime}\right) \\
& \left(-\beta^{\prime}-\gamma^{\prime}-\rho^{\prime}-\varsigma_{1} k_{1}-\ldots-\varsigma_{t} k_{t} ; u_{1}^{\prime}, \ldots, u_{r}^{\prime}, \frac{0, \ldots, 0}{s}, t_{1}^{\prime}, \ldots, t_{s}^{\prime}\right),\left(1+\sigma_{1}^{\prime}+\tau_{1}^{\prime} k_{1}+\ldots+\tau_{1}^{(t)} k_{t} ; w_{1}^{\prime}, \ldots, w_{1}^{(r)}, \frac{0, \ldots, 0}{s}, 1, \frac{0, \ldots, 0}{s-1}\right), \ldots, \\
& \left(-\alpha^{\prime}-\gamma^{\prime}-\rho^{\prime}-\varsigma_{1} k_{1}-\ldots-\varsigma_{t} k_{t} ; u_{1}^{\prime}, \ldots, u_{r}^{\prime}, \frac{0, \ldots, 0}{s}, t_{1}^{\prime}, \ldots, t_{s}^{\prime}\right),\left(1+\sigma_{1}^{\prime}+\tau_{1}^{\prime} k_{1}+\ldots+\tau_{1}^{(t)} k_{t} ; w_{1}^{\prime}, \ldots, w_{1}^{(r)}, \frac{0, \ldots, 0}{2 s}\right), \ldots, \\
& \left(1+\sigma_{1}^{\prime}+\tau_{1}^{\prime} k_{1}+\ldots+\tau_{1}^{(t)} k_{t} ; w_{1}^{\prime}, \ldots, w_{1}^{(r)}, 1, \frac{0, \ldots, 0}{2 s-1}\right) \\
& \left(1+\sigma_{s}^{\prime}-\tau_{s}^{\prime} k_{1}+\ldots+\tau_{s}^{(t)} k_{t} ; w_{1}^{\prime}, \ldots, w_{1}^{(r)}, \frac{0, \ldots, 0}{2 s}\right) \text {, } \\
& \left.\left(d_{j}^{\prime}, \delta_{j}^{\prime}\right)_{1, Q_{1}} ; \ldots ;\left(d_{j}^{(r)}, \delta_{j}^{(r)}\right)_{1, Q_{r}} ; \frac{(\mathrm{O}, 1), \ldots,(\mathrm{O}, 1)}{2 s}\right]
\end{aligned}
$$

Provided that

$$
\operatorname{Re}(\alpha)>0
$$

the

quantities $t_{1}, t_{1}^{\prime} \ldots, t_{s}, t_{s}^{\prime}, \lambda_{1} \eta_{1}^{\prime}, \ldots, \lambda_{t} \eta_{1}^{(t)}, \ldots, \lambda_{1} \eta_{s}^{\prime}, \ldots, \lambda_{t} \eta_{s}^{(t)}, u_{1}, v_{1}^{\prime}, \ldots, v_{s}^{\prime}, u_{r}, v_{1}^{(r)}, \ldots, v_{s}^{(r)}, \varsigma_{1} \tau_{1}^{\prime}, \ldots, \varsigma_{s} \tau_{s}^{\prime}, w_{1}^{\prime}, \ldots, w_{s}^{\prime}, w_{1}^{(r)}, \ldots, w_{s}^{(r)}$ are all positive (some of them may however decrease to zero provided that the resulting integral has a meaning),

(ii) $\operatorname{Re}(\rho)+\sum_{i=1}^{r} u_{i} \min _{1 \leq j \leq M_{i}}\left[\operatorname{Re}\left(\frac{d_{j}^{(i)}}{\delta_{j}^{(i)}}\right)\right]+1>0$

And $\operatorname{Re}\left(\rho^{\prime}\right)+\sum_{i=1}^{r} u_{i} \min _{1 \leq j \leq M_{i}}\left[\operatorname{Re}\left(\frac{d_{j}^{(i)}}{\delta_{j}^{(i)}}\right)\right]+1>0$

Proof of (8): To prove the fractional derivative formula (FDF)1, we first express the product of a general class of polynomials occurring on its left-hand side in the series form given by (4), replace the multivariable $A$ function occurring theorem by its well known Mellin-Barnes contour integralgiven by (5), interchange the order of summations, $\left(\xi_{1}, \ldots, \xi_{r}\right)$-integrals and taking the fractional derivative operator inside (which is permissible 
under the conditions stated with (8)) and make a little simplification. Next, we express the terms $\left(x^{t_{1}}+\alpha_{1}\right)^{\sigma_{1}+\eta_{1}^{\prime} k_{1}+\ldots+\eta_{1}^{(t)} k_{t}-v_{1}^{\prime} \xi_{1}-\ldots-v_{1}^{(r)} \xi_{r}}, \ldots,\left(x^{t_{s}}+\alpha_{s}\right)^{\sigma_{1}+\eta_{s}^{\prime} k_{1}+\ldots+\eta_{s}^{(t)} k_{t}-v_{s}^{\prime} \xi_{1}-\ldots-v_{s}^{(r)} \xi_{r}}$ so obtained in terms of MellinBernes contour integral ([16]. P.18, eq. (2.6.4); p.10, eq.(2.1.1)). Now, interchanging the order of $\left(\xi_{r+1}, \ldots, \xi_{r+s}\right)$ and $\left(\xi_{1}, \ldots, \xi_{r}\right)$-integrals (which is also permissible under the conditions stated with (8)), and evaluating the $\mathrm{x}$-integral thus obtained by using the unknown formula ([10], p.16 Lemma 1)

$I_{0, x}^{\alpha, \beta, \gamma}\left[x^{\lambda}\right]=\frac{\Gamma(1+\lambda) \Gamma(1-\beta+\gamma+\lambda)}{\Gamma(1-\beta+\lambda) \Gamma(1+\alpha+\gamma+\lambda)} x^{\lambda-\beta}$

$\operatorname{Re}(\lambda)>0=\max [0, \operatorname{Re}(\beta-\gamma)]-1$

And reinterpreting the multivariable Mellin-Barnes contour integral so obtained in terms of the $A$-function of $r+s$ variables, we easily arrive at the desired formula (8) after a little simplification.

Proof of (9): To prove FDF 2, we take

$$
f(x)=x^{\rho} \prod_{i=1}^{x-1}\left(x^{t_{i}}+\alpha_{i}\right)^{\sigma_{i}} A^{*}\left[z_{r+1} x^{u_{r}+1} \prod_{i=1}^{s-1}\left(x^{t_{i}}+\alpha_{i}\right)^{-v_{i}^{(r+1)}}, \ldots, z_{r+r} x^{u_{r}+r} \prod_{i=1}^{s-1}\left(x^{t_{i}}+\alpha_{i}\right)^{-v_{i}^{(r+r)}}\right]
$$

And

$$
\begin{aligned}
& g(x)=\left(x^{t_{s}}+\alpha_{s}\right)^{\sigma_{s}} \prod_{j=1}^{t} S_{n_{j}}^{m_{j}}\left[e_{j} x^{\lambda_{j}} \prod_{i=1}^{s}\left(x^{t_{i}}+\alpha_{i}\right)^{\eta_{i}^{(j)}}\right] \\
& A\left[z_{1} x^{u_{1}} \prod_{i=1}^{s}\left(x^{t_{i}}+\alpha_{i}\right)^{-v_{i}}, \ldots, z_{r} x^{u_{r}+r} \prod_{i=1}^{s}\left(x^{t_{i}}+\alpha_{i}\right)^{-v_{i}^{(r)}}\right]
\end{aligned}
$$

In the left-hand side of (9); and apply the following generalized Leibnitz rule for the fractional integrals

$$
I_{0, x}^{\alpha, \beta, \gamma}\{f(x) g(x)\}=\sum_{t=0}^{\infty}\left(\begin{array}{l}
-\beta \\
t
\end{array}\right) I_{0, x}^{\alpha, \beta-l, \gamma}\{f(x)\} I_{0, x}^{\alpha, \beta-l, \gamma}\{g(x)\}
$$

We easily obtain FDF 2 after a little simplification on making use of FDF 1 and known result ([5], p. 91, eq. (6)).

Proof of (11): To prove FDF 3, we use the formula FDF 1 twice with respect to the variable $y$, and then with respect to the variable $x$; here $x$ and $y$ are independent variables.

\section{Special Cases and Applications}

The fractional derivative formulae 1,2 and 3 established here are unified in nature and act as key formulae. Thus the general class of polynomials involved in FDF 1,2 and 3 reduce to a large spectrum of polynomials listed by Srivastava and Singh ([18], pp.158-161), and so from formula 1,2 and 3 we can further obtain various fractional derivative formulae involving a number of simpler polynomials. Again, the multivariable $A$-function occurring in these formulae can be suitably specialized to a remarkably wide variety of useful functions (or product of several such functions) which are expressible in terms of $E, F, G$ and $H$ functions of one and more variables. For example, if $M=N=P=Q=0$, the multivariable $A$-function occurring in the left-hand side of these formulae would reduce immediately to multivariable $H$-function due to Srivastava et. al.[16]. Thus the various special cases of the multivariable $H$-function can be used to derive from these fractional derivative formulae a number of other FDF involving any of these simpler special functions.

On reducing the operator defined by (1) to the Riemann-Liouville operator given by (2), we arrive at three fractional derivative formulae involving these operators but we do not record them here explicitly. Again , our FDF 1,2 and 3 will also rise in essence to a number of other FDF lying scattered in the literature (see [14], pp. 563-564, eqs. 92.1)-(2.3), [15], pp. 644-645, eqs. (2.1)-(2.3), [4], pp. 71-72, eq. (2.1) and [3], p. 171, eq. (3.1)) on making suitable substitutions.

Also, if we take $M=N=P=Q=0, \sigma_{i}=0=v_{i}^{\prime}=\ldots=v_{i}^{(r)}, i=1,2, \ldots, s$ and $n_{j}=0, j=1, \ldots, t$ in (8) (the polynomials $S_{0}^{m_{1}}, \ldots, S_{0}^{m_{t}}$ will reduce to $A_{0,0}^{\prime}, \ldots, A_{0,0}^{(t)}$ respectively which can be taken to be unity without loss of generality), we arrive at the formula given by ([11], p.532, eq. (4.1)).

If in FDF 1 , we take $M=N=P=Q=0, t=2$ and reduce the polynomial $S_{n_{1}}^{m_{1}}$ to the Hermite polynomial ([18], p. 158, eq. 91.4)), the polynomial $S_{n_{2}}^{m_{2}}$ to the Leguerre polynomial ([18], p.159, eq. (1.8)), the 
multivariable $I$-function to the product of $r$ different Whittakar functions ([16], p.18, eq. (2.6.7)), we arrive at the following new and interesting special case of the FDF 1 after a little simplification

$$
\begin{aligned}
& I_{0, x}^{\alpha, \beta, \gamma}\left\{x^{\rho+\sum_{l=1}^{r} b_{l}+\frac{n_{l}}{2}} \prod_{i=1}^{s}\left(x^{t_{i}}+\alpha_{i}\right)^{\sigma_{i}} H_{n_{l}}\left\{\frac{1}{2 \sqrt{x}}\right\} \boldsymbol{L}_{n_{2}}^{(\theta)}(x) \prod_{l=1}^{r}(\exp )^{-\frac{z}{2} x} W_{\mu_{l}, \nu_{l}}\left(z_{l} x\right)\right\} \\
& =\frac{\prod_{l=1}^{r}\left(z_{l}\right)^{-b_{l}} \alpha_{1}^{\sigma_{1}} \ldots \alpha_{s}^{\sigma_{s}} x^{\rho-\beta}}{\Gamma\left(-\sigma_{1}\right) \ldots \Gamma\left(-\sigma_{s}\right)} \sum_{k_{1}=0}^{\left[n_{1} / 2\right]} \sum_{k_{2}=0}^{\left[n_{2}\right]} \frac{\left(-n_{1}\right)_{2 k_{1}}\left(-n_{2}\right)_{k_{2}}}{k_{1} ! k_{2} !}(-1)^{k_{1}}\left(\begin{array}{c}
n_{2}+\theta \\
n_{2}
\end{array}\right) x^{k_{1}+k_{2}}
\end{aligned}
$$

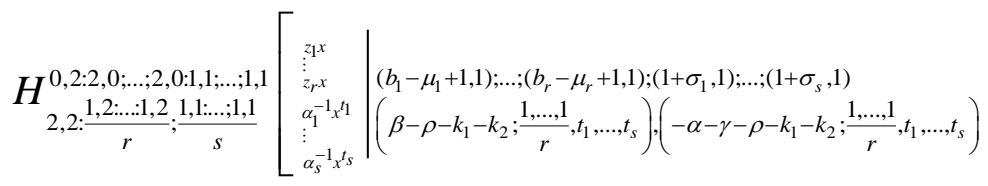

$$
\begin{aligned}
& \left.\begin{array}{l}
\left(-\rho-k_{1}-k_{2} ; 1, \ldots, 1, t_{1}, \ldots, t_{s}\right),\left(\beta-\gamma-\rho-k_{1}-k_{2} ; 1, \ldots, 1, t_{1}, \ldots, t_{s}\right) \\
\left(b_{1} \pm v_{1}+\frac{1}{2}, 1\right) ; \ldots ;\left(b_{r} \pm v_{r}+\frac{1}{2}, 1\right) ; \frac{(0,1) ; \ldots ;(0,1)}{s}
\end{array}\right]
\end{aligned}
$$

The conditions of validity of (14) can be easily obtained from those of (8).

Several other interesting and useful special cases of our main fractional derivative formulae 1,2 and 3 involving the product of a large variety of polynomials (which are special cases of $S_{n_{1}}^{m_{1}}, \ldots, S_{n_{t}}^{m_{t}}$ ) and numerous simple special functions involving one or more variables (which are particular cases of the multivariable $A$-function) can also be obtained but we do record them here for lack of space.

\section{References}

[1] Fox, C.; The $G$ and $H$-functions as symmetrical Fourier kernels, Trans. Amer.Math. Soc. 98 (1961), 395-429.

[2] Gautam, B.P., Asgar, A.S. and Goyal, A.N.; On the multivariable $A$-function, Vijnana Parishad Anusandhan Patrika, vol. 29(4), 67-81.

[3] Gupta, K.C. and Agarwal, S.M.; Fractional integral formulae involving a general class of polynomials and the multivariable $H$ function, Proc. Indian Acad. Sci. (Math. Sci) 99 (1989), 169-173.

[4] Gupta, K.C. , Agarwal, S.M. and Soni, R.C.; Fractional integral formulae involving the multivariable $H$-function and a general class of polynomials, Indian J. Pure Appl. Math. 21(1990), 70-77.

[5] Gupta, K.C. and Soni, R.C.; A study of $H$-functions of one and several variables, J. Rajastahan Acad. Phys. Sci. 1(2002), 8994.

[6] Mathai A.M. and Saxena, R.K.; The $H$-function with Applications in Statistics and Other Disciplines,John Wiley \& Sons, New Delhi(1978).

[7] Miler, K.S. and Ross, B.; An introduction to the fractional calculus and fractional differential equations, John-Wiley \& Sons, (1993).

[8] Oldham, K.B. and Spanier, J.; The fractional Calculus, Academic Press, NewYork (1974).

[9] Saigo, M.A. ; A remark on integral operators involving the Gauss hypergeometric functions, Math. Rep. Kyunshu. Univ. 11(1978), 135-143.

[10] Saigo, M and Raina, R.K.; Fractional calculus operators associated with a general class polynomials, Fukuoka Univ. Reports 18(1988), 15-22.

[11] Saigo, M and Raina, R.K.; Fractional calculus operators associated with the $H$-function of several variables,in:Analysis, Geometry and Groups: A Riemann Legacy Volume, (eds) H.M. Srivastava and Th M Rassias (Palm Harbor, Florida 34682-1577, USA)(Hadronic Press) ISBN 0-911767-59-2 (1993) 527-538.

[12] 12.Soni, R.C. and Singh, D.; Certain fractional derivative formulae involving the product of a general class of polynomials and the multivariable $H$-function, Proc. Indian Acad. Sci. (Math. Sci.) vol. 112 (4), (2002), 551-562.

[13] Srivastava, H.M.; A contour integral involving Fox's $H$-function, Indian J. Math. 14(1972), 1-6.

[14] Srivastava, H.M., Chandel, R.C. and Vishwakarma, P.K.; Fractional derivatives of certain generalized hypergeometric functions of several variables, J. Math. Anal. Appl. 184 (1994), 560-572.

[15] Srivastava, H.M. and Goyal, S.P.; Fractional derivatives of the $H$-function of several variables, J. Math. Anal. Appl. 112 (1985), 641-651.

[16] Srivastava, H.M.,Gupta, K. C. and Goyal, S. P.: The H-function of One and Two Variables with Applications. South Asian Publishers, New Delhi (1982).

[17] Srivastava, H.M. and Panda, R.; Some bilateral generating functions for a class of generalized hypergeometric polynomials, J. Reine. Angew Math. 283/284(1976), 265-274.

[18] Srivastava, H.M. and Singh, N.P.; The integration of certain products of the multivariable $H$-function with a general class of polynomials, Rend. Circ. Mat. Palermo, 32 (1983), 157-187. 